\title{
CHARACTERISING THE MUCOSAL IMMUNE RESPONSE IN THE GREATER AMBERJACK (SERIOLA DUMERILI)
}

\author{
Douglas Milne $^{1}$, Steve Bird ${ }^{2}$, Daniel Montero ${ }^{3}$, Alvaro Fernández-Montero ${ }^{3}$, Felix Acosta $^{3}$ \& \\ Chris J. Secombes ${ }^{1}$
}

${ }^{I}$ Scottish Fish Immunology Research Centre, Institute of Biological and Environmental Sciences, University of Aberdeen, Tillydrone Avenue, Aberdeen, AB24 2TZ, UK

${ }^{2}$ Molecular Genetics, Department of Biological Sciences, University of Waikato, Hamilton, New Zealand.

${ }^{3}$ Grupo de Investigación en Acuicultura, Insituto Ecoaqua. Universidad de Las Palmas de Gran Canaria, Canary Islands, Spain

Production of the greater amberjack (Seriola dumerili) is increasing rapidly in European aquaculture. However this rapid increase in production has highlighted bottlenecks in several aspects of their rearing, including knowledge of their susceptibility to infection. Indeed, currently little is known about the immune system and immune response in this species which severely limits approaches for disease control, should widespread disease outbreaks arise. Therefore, this project aims to characterise the immune response in the greater amberjack, with an emphasis on mucosal immunity, following stimulation with PAMPs and bacterial/parasite infection. At the beginning of this project there were no immune genes that had been sequenced for the greater amberjack, which made analysis of the immune response problematic. Therefore, before analysis of the immune response could begin, key mucosal immune genes had to be identified and sequenced, facilitating the development of QPCR primers which allowed the monitoring of gene expression. This was achieved by utilising the sequences of these genes in closely related species, such as yellowtail amberjack (Seriola lalandi), as well as cloning using primers to conserved domains. Once these primers had been developed the response of greater amberjack to the PAMPs poly:IC, LPS and flagellin was studied in vitro and in vivo. This preliminary interrogation of the greater amberjack immune responses will pave the way for future experiments to study the impact of dietary treatments on expression of key immune molecules as well as study of the immune response to pathogen infection.

This project has received funding from the European Union's Seventh Framework Programme for research, technological development and demonstration (KBBE-2013-07 single stage, GA 603121, DIVERSIFY).

Key words

Greater Amberjack, immune responses, mucosal immunity, expression analysis.

§Corresponding author. Tel.: +441224 272827.

E-mail address: c.secombes@abdn.ac.uk 